\title{
Co-design of a culturally-tailored diet \& lifestyle intervention for diabetes management in the UK African-Caribbean community
}

\author{
A.P. Moore ${ }^{1}$, S.H. Stanton-Fay ${ }^{2}$, C.A. Rivas ${ }^{3}$, S. Harding ${ }^{1}$ and L.M. Goff ${ }^{1}$ \\ ${ }^{1}$ Diabetes \& Nutritional Sciences Division, King's College London, \\ ${ }^{2}$ Research Dept. of Clinical, Education \& Health Psychology, University College London and \\ ${ }^{3}$ Social Sciences Research Unit, University College London
}

Education promoting healthy eating, weight management, and increased physical activity, forms the cornerstone of type 2 diabetes (T2D) management, however, cultural barriers may limit healthcare access and contribute to poorer outcomes for patients of African-Caribbean (AfC) ethnicity. Culturally-tailored interventions are an effective means by which to address such inequalities and it is recommended that interventions are underpinned by appropriate theory ${ }^{(1)}$. The Capability-Opportunity-Motivation (COM-B) behaviour change framework provides intervention designers with a systematic methodology to identify key target behaviours, select appropriate intervention functions and link these to evidence-based behaviour change techniques ${ }^{(2)}$. This research aims to use co-design methods and the COM-B framework to develop a culturally-tailored diet and lifestyle intervention for managing T2D in AfC patients.

Focus groups were held with AfC T2D patients and were stratified by gender and ethnicity. Groups, held in the community and on university premises, lasted 2-hrs and were digitally recorded and transcribed. Barriers and facilitators to the adoption of diet, physical activity and weight advice were coded independently by two researchers, using the COM-B framework.

Eleven focus groups with 41 patients (68\% female; $58 \%$ African, $42 \%$ Caribbean; $89 \%$ first generation UK immigrants; mean age $62 \mathrm{yrs}$ ) were conducted. The following themes were identified and mapped to the COM-B domains:

Capability (knowledge, skills): Barriers exist around the application of dietary advice to the cultural foods commonly consumed and in understanding of the physical activity guidance. Hectic lives, involving multiple jobs and caring responsibilities suggest support is needed to develop capability around goal setting and action planning.

Opportunity (social, environmental): Social opportunity for patients to follow guidance is reduced by cultural identity being strongly associated with large portion sizes of traditional carbohydrate foods. An acceptance of larger body shapes and cultural associations of weight loss with ill health, influences attitudes towards weight management. Body Mass Index targets are rejected as inappropriate for the Black body shape.

Motivation (beliefs, intentions, emotions): Patients are strongly motivated by the desire to avoid the complications of T2D and a focus on health consequences could support motivation for change. Collectivism and positive role models are also central facilitators to encourage change. Walking and dancing as means to exercise resonated well with cultural habits.

Behavioural change techniques to educate, enable, persuade and model were selected and will include AfC patient stories of success delivered by video, cook and taste sessions, use of graded physical activity tasks and practical activities to improve self-efficacy in making appropriate dietary choices.

The application of the COM-B framework demonstrates that while African and Caribbean T2D patients may be strongly motivated to manage their T2D to avoid long term health consequences, an intervention needs to support their ability to adapt healthcare information to cultural habits. Engaging social support structures and role models within the community may increase opportunity and motivation to adopt recommended health behaviours.

Funded by the National Institute of Health Research (NIHR)

1. Liu J, Davidson E, Bhopal R, White M, Johnson M, Netto G et al. (2012) Health Technol Assess 16, 1-469.

2. Michie S, van Stralen MM, West R (2011) Implement Science 6, 42. 